\title{
La Atención a la Diversidad Funcional en Educación Primaria
}

\section{Attention to the Functional Diversity on Primary Education}

\author{
Virginia Fuentes, Marta García-Domingo *, Patricia Amezcua Aguilar, Teresa Amezcua \\ Universidad de Jaén, España
}

\section{DESCRIPTORES: \\ Derecho a la educación \\ Educación universal \\ Estrategias educativas \\ Necesidades \\ educacionales \\ Discriminación \\ educacional}

\section{RESUMEN}

Pese a los múltiples estudios que confirman la pertinencia de dar valor a la diversidad presente en las aulas a través de estrategias orientadas a la educación inclusiva todavía encontramos manifestaciones evidentes de segregación escolar. El presente estudio, a través de la técnica del Grupo Focal, identifica apoyos y obstáculos presentes en el ámbito de educación primaria que percibe el equipo educativo. Para ello se analizan cuestiones vinculadas al imaginario social de la diversidad, actitudes, demandas, mitos, prejuicios y limitaciones tanto internos como externos, entre otros. Destaca la tendencia a vincular el concepto de diversidad a la diversidad funcional (que marca el resto del análisis de la realidad del presente estudio), la dificultad de adaptar la heterogeneidad en los ritmos de aprendizaje a la homogeneización de resultados académicos exigidos, la preponderancia de contenidos formales frente a los transversales y una burocracia que incentiva a las adaptaciones curriculares formales. En la comunidad educativa, identifican situaciones de rechazo y actitudes discriminatorias transmitidas por parte de los familiares de alumnado normotípico sobre la diversidad funcional y una implicación insuficiente en la consolidación de espacios escolares inclusivos en un porcentaje considerable del profesorado. Se requiere, por tanto, una puesta en valor de la diversidad y de los beneficios de la inclusión para el conjunto de la sociedad.

\section{KEYWORDS:}

Right to education

Universal education

Educational strategies

Educational needs

Educational discrimination

\section{ABSTRACT:}

Despite the multiple studies that confirm the relevance of placing value on classrooms diversity through strategies oriented to inclusive education, we still find evident manifestations of school segregation. This study, using the Focus Group technique, identifies supports and obstacles in the field of primary education, that are perceived by educational team. For this, issues related to social imaginary of diversity, attitudes, demands, myths, prejudices and limitations, both internal and external, among others, are analysed. The tendency to link the concept of diversity to functional diversity stands out (which marks the rest of the analysis of the reality of this study), the difficulty of adapting heterogeneity in learning rhythms to the homogenization of academic results required, the preponderance of formal content over cross-cutting and a bureaucracy that encourages formal curricular adaptations. In the educational community, they identify situations of rejection and discriminatory attitudes transmitted by the relatives of norm-typical students regarding functional diversity and an insufficient implication in the consolidation of inclusive school spaces in a considerable percentage of the teaching staff. Therefore, it is necessary to place value on diversity and on the benefits of inclusion for society as a whole.

CÓMO CITAR:

Fuentes, V., García-Domingo, M., Amezcua Aguilar, P. y Amezcua, T. (2021). La atención a la diversidad funcional en educación primaria. REICE. Revista Iberoamericana sobre Calidad, Eficacia y Cambio en Educación, 19(1), 91-106. https://doi.org/10.15366/reice2021.19.1.006

*Contacto: mgdoming@ujaen.es

ISSN: $1696-4713$

www.rinace.net/reice/

revistas.uam.es/reice
Recibido: 2 de abril 2020

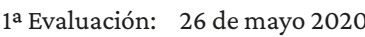

2a Evaluación: 27 de junio 2020

Aceptado: $\quad 7$ de julio 2020 


\section{Introducción}

El reconocimiento del acceso a la educación como un derecho humano no es baladí. A través de la educación no sólo se facilita a las personas el aprendizaje de conocimientos, sino también la integración de los valores sociales y culturales de la comunidad de referencia y la adquisición de habilidades y destrezas que capacitan para el desarrollo de una vida social plena y el reconocimiento de la propia dignidad. Como contrapartida, la multiplicidad de potencialidades de la educación implica que su aplicación práctica responda a procesos complejos con diferentes mecanismos de articulación. En este trabajo nos referiremos a dos procedimientos a los que se recurre desde las instituciones para la implementación de la educación: la segregación escolar y la inclusión educativa. Se trata de dos vías opuestas de estrategia educativa que se sustentan en posiciones ideológicas antagónicas en términos de justicia social.

Siguiendo a Murillo y Duk (2016), la segregación escolar es "la distribución desigual de los estudiantes en las escuelas en función de sus características personales o procedencia social y cultural” (p.11). La fundamentación teórica del segregacionismo escolar sitúa la diferencia como elemento disruptivo del proceso educativo en la escuela ordinaria. Merle (2012) identifica cuatro dimensiones de segregación escolar: 1) segregación por sexo, 2) segregación étnica, 3) segregación académica, y 4) segregación social. Estas cuatro dimensiones se interrelacionan y tienen en buena medida como trasfondo la variable económica, dando lugar a situaciones de desigualdad y exclusión social.

En contraposición, el paradigma de la Educación Inclusiva surge como respuesta a las desigualdades en materia de derechos humanos que devienen en situaciones de injusticia social en el ámbito de la educación (UNESCO, 1994). Este modelo educativo responde a necesidades identificadas desde las tres principales conceptualizaciones actuales de Justicia Social: Justicia como distribución, Justicia como Reconocimiento y Justicia como Participación (Murillo y Hernández, 2011). Las tres perspectivas teóricas analizan los procesos de desigualdad desde diferentes dimensiones de la realidad social que, en el ámbito educativo, confluyen en los procesos finales de segregación escolar. Como señala Rubia (2013) "la segregación étnica, académica y social correlacionan claramente entre ellas” (p. 49), ya que las posiciones sociales están determinadas por las posiciones en el mercado de trabajo y estas a su vez por las capacidades funcionales, ya sea físicas, psíquicas, cognitivas, culturales o emocionales.

La perspectiva teórica que identifica Justicia social como Distribución argumenta que el origen de las desigualdades se encuentra en la existencia de una estructura socioeconómica injusta que reproduce dinámicas excluyentes. Estas desigualdades se hacen patentes en la segregación escolar de las clases y grupos sociales, generalmente vinculadas también con la segregación espacial. La equidad se logra con la redistribución de los recursos o "bienes primarios" que son causa de desigualdad (Rawls, 1971, 2002). Sin embargo, esta redistribución de bienes se lleva a cabo generalmente a partir de medidas compensatorias que, en muchas ocasiones, vuelven a reproducir la segregación escolar socioeconómica.

Por su parte, la Justicia entendida como Reconocimiento (Fraser, 2008; Fraser y Honneth, 2005) pone el foco en la erradicación de la dominación cultural y el respeto a la diferencia como ejes vertebradores de la desigualdad. Las diferencias basadas en constructos sociales como el género, la cultura o la discapacidad son los principales ejes de estas formas de injusticia. La segregación escolar en este sentido reproduce "injusticias culturales vinculadas a procesos de representación, interpretación y comunicación” (Murillo y Hernández, 2011, p. 17). Las medidas correctoras deben centrarse por tanto en el desvelamiento de prejuicios y estereotipos, la visibilización y puesta en valor de las identidades colectivas normoatípicas, y la promoción del respeto a la diferencia. La segregación por sexo ha sido la dimensión más visibilizada en los espacios políticos de toma de decisiones de las últimas décadas en España y la que de forma más evidente ejemplifica las imposiciones de la cultura dominante. Sin embargo, son la segregación étnica o cultural y la segregación socioeconómica las que arrojan datos más alarmantes y con más repercusiones negativas para la consecución de la calidad de la educación para todos (Murillo y Martínez-Garrido, 2017; Murillo, Martínez-Garrido y Belavi, 2017). También la segregación académica por razón de discapacidad está estrechamente vinculada a la dominación cultural de la sociedad normotípica, que niega el reconocimiento de la diversidad funcional como un elemento enriquecedor de la comunidad educativa y de la sociedad. La teoría de las capacidades humanas (Nussbaum, 2012; Sen, 2010) señala la necesidad de entender al individuo desde sus capacidades diversas como una nueva forma de dimensionar al ser humano en sociedad. Sin embargo, la segregación del alumnado con discapacidad se institucionaliza ya con la diferenciación entre 
la educación general y la educación especial, y más tarde, con trayectorias de escolarización basadas en el rendimiento académico como el "agrupamiento homogéneo" -que permite adaptar el currículo al nivel de cada grupo del centro educativo- (Rubia, 2013) o los Programas de Diversificación, de Garantía Social y de Cualificación Profesional Inicial (PCPI).

Finalmente, la Justicia como Participación (Young, 2000, 2002) da un paso más allá en estas interpretaciones planteando que la consecución de la equidad, ya sea de bienes y recursos o de reconocimiento de la diferencia, pasa por garantizar el acceso a los procesos de toma de decisiones en las instituciones. En este sentido los grupos sociales que no gozan de respeto y reconocimiento social son discriminados y excluidos de los procesos de toma de decisiones que tienen que ver con sus propios intereses. La participación, como dinámica social de ciudadanía, requiere de un aprendizaje para ser ejercida, por lo que los colectivos excluidos se ven inmersos en dinámicas de exclusión que se retroalimentan. En este sentido, el sistema educativo, como medio de aprendizaje de competencias sociales, es el campo de entrenamiento del ejercicio de ciudadanía activa y participativa, y la Educación Inclusiva el paradigma desde el que se quiere hacer efectiva la equidad en las aulas. La inclusión educativa como participación pone el foco en la escolarización ordinaria de todo el alumnado como vía de democratización del conocimiento y el aprendizaje. La universalización de la escolarización en las escuelas ordinarias debe incorporar tres aspectos fundamentales: el respeto a la diversidad, la adaptación a las características individuales y/o grupales, sus necesidades educativas y el derecho a la participación.

\section{La educación inclusiva}

El germen del paradigma de la Educación Inclusiva es la Conferencia Mundial de Educación para Todos de la UNESCO de 1990 (Dussan, 2010) donde se plantea la necesidad de incorporar la Educación Especial al sistema de enseñanza como modelo de intervención educativa con alumnado con Necesidades Educativas Especiales (NEE), entendiéndose éstas como las dificultades o limitaciones derivadas de la discapacidad que éste pueda presentar puntual o procesualmente durante su proceso de enseñanza-aprendizaje (Luque-Parra y Luque-Rojas, 2013). Para ello, se ponen en marcha "estrategias que posibiliten una auténtica igualdad de oportunidades" (UNESCO, 1994, p. 11). No obstante, la educación inclusiva no se plantea como destinatarios exclusivamente a personas con NEE de tipo funcional, sino que se dirige a todo el alumnado en situación de exclusión educativa por cualquier razón (étnica, religiosa, de género o/y económica) y que debido a ello requiera de atención educativa distinta a la ordinaria. El objetivo de la inclusión se centra desde esta perspectiva en atender al alumnado con necesidades específicas de apoyo educativo (NEAE) derivadas tanto de las dificultades de aprendizaje como de las altas capacidades intelectuales o debidas a situaciones personales, sociales o familiares como la escolarización tardía o la deprivación. En este sentido, el paradigma de la educación inclusiva entiende la escuela ordinaria como eje vertebrador de un modelo de sociedad que no sólo respete la diferencia, sino que la incluye y la pone en valor.

A nivel operativo el modelo de Educación Inclusiva supone la integración del sistema general de educación y el de educación especial en un sistema unitario (Araque y Barrio, 2010). Bajo el principio de normalización, la escuela inclusiva se plantea como un modelo educativo integrador de la diversidad humana que debe ser capaz de dar respuesta a las distintas necesidades que plantea cada individuo en su proceso educativo. Cada niño y niña tiene unas características, intereses, capacidades y necesidades de aprendizaje propios, por lo que los programas educativos han de diseñarse e implementarse articulando todos estos elementos de diversidad (Arnaiz, 2012). La efectividad de este modelo requiere conjugar las actuaciones institucionales y la promoción de la participación del alumnado, entendiendo que el currículo educativo ha de adaptarse a las capacidades de éste y no al contrario (García-Domingo, Amezcua, y Fuentes, 2019; Garzón, Calvo y Orgaz, 2016; Martínez, De Haro y Escarbajal, 2017).

A este respecto, uno de los mayores retos de la escuela se encuentra en compatibilizar la consecución de los objetivos curriculares marcados por las normativas educativas y la atención a las necesidades individuales que la diversidad del alumnado requiere. Para la armonización de los principios de calidad de la educación e igualdad de oportunidades los centros educativos cuentan con el "plan de atención a la diversidad" como instrumento operativo en el que se recogen las medidas ordinarias y extraordinarias que forman parte de su proyecto educativo y entre las que se encuentra la adaptación curricular (Araque y Barrio, 2010; Arnaiz, 2012) y el desarrollo de competencias transversales. Las necesidades que plantea la diversidad funcional 
del alumnado fuerza al profesorado a diseñar nuevas estrategias en el proceso de enseñanza-aprendizaje y desligarse de los patrones más constreñidos de la escuela tradicional. Las dinámicas innovadoras finalmente permiten que todo el alumnado pueda acceder a un aprendizaje significativo (Arnaiz, 2012). Sin embargo, como apuntan Garzón, Calvo y Orgaz (2016, p. 27) "las actitudes de toda la comunidad educativa, en concreto las actitudes del profesorado, son claves para atender la diversidad del alumnado". Distintos autores señalan que las actitudes del profesorado hacia la inclusión en el aula han de analizarse desde los tres elementos que componen este concepto: 1) el componente cognitivo, relacionado con los conocimientos y creencias; 2) el componente afectivo, basado en sentimientos y preferencias; y 3) el componente conductual, relativo a las acciones que esto llevan a cabo (Coll et al., 1994; Díaz, 2006; Garzón, Calvo y Orgaz, 2016)

En este trabajo nos centraremos en analizar el papel determinante de las aulas, familias y contexto social en la inclusión del alumnado con NEE. De especial relevancia en este sentido son las percepciones de los/ as maestros/as respecto a la inclusión del alumnado con diversidad funcional y los beneficios que su escolarización en la escuela ordinaria conlleva para la comunidad educativa.

\section{Método}

El objetivo de esta investigación es analizar la concepción y gestión de la diversidad presente en las aulas de educación primaria, así como indagar en los apoyos y obstáculos que encuentran los/as maestros/as de centros públicos a la educación inclusiva. Para ello se diseñó una metodología cualitativa basada en la técnica del Grupo Focal como instrumento procedimental más idóneo para la "identificación de necesidades personales y comunitarias" (Escobar y Bonilla-Jiménez, 2017, p. 53).

En todo momento se fomentó la libertad de los participantes para el desarrollo y exposición de sus sensaciones y percepciones, conduciéndose no obstante los discursos hacia temas de interés para la investigación a través de la figura de la moderadora. Para la selección del perfil de participantes se atendió a criterios de representatividad y proporcionalidad, con la pretensión de que los distintos discursos y perspectivas sobre la temática abordada tuvieran cabida. Sin embargo, se optó por centrar la muestra en el ámbito público, ya que el objeto del estudio era indagar en la gestión que se hace de la diversidad en centros públicos de educación primaria.

Se realizaron dos grupos focales, con un total de 16 profesionales de educación primaria (31,25\% hombres y $68,75 \%$ mujeres), estando representadas las distintas figuras profesionales presentes en los centros y que dan respuesta a las necesidades diversas. Si bien en un planteamiento inicial de la presente investigación se contempló la opción de incluir en la muestra a miembros de equipos directivos de centros educativos, finalmente y atendiendo al objeto de estudio, se optó por recoger exclusivamente el discurso de los docentes implicados en la atención directa de alumnado con NEE. Cabe destacar que todos los informantes desarrollan su labor profesional en centros públicos de la Provincia de Jaén (Comunidad Autónoma Andaluza), integrados tanto en municipios de menos de 100.000 habitantes (56,25\% de los participantes) como en distintos centros de la capital de provincia, que cuenta con una población superior a 100.000 habitantes $(43,75 \%)$. Se recogen, asimismo, las percepciones de maestros que desarrollan su labor tanto en centros ordinarios $(68,75 \%)$, como en centros preferentes $(31,25 \%)$, contando en algunos casos los/as informantes con una trayectoria profesional que incluye tanto a los primeros como a los segundos.

El perfil profesional de los sujetos participantes fue: maestros/as de aulas ordinarias de escuelas públicas de primaria, maestros/as de aulas de apoyo a la integración, pedagogo/a, auxiliar técnico educativo y maestros/as con algún tipo de formación específica en el tema. Con respecto a los años de experiencia profesional, un $56,25 \%$ contaban con entre 1 y 5 años de experiencia y un $43,75 \%$ con entre 6 y 15 años. Los grupos fueron conformados de forma mixta, de manera que, en cada uno, hubiese representación de todas las figuras profesionales implicadas. El objetivo de esto fue generar un discurso participado, resultado de la interacción de postura y opiniones.

El desarrollo de los grupos se realizó en una sala dotada de una mesa central y sillas móviles, contándose con una persona moderadora y una persona asistente a la moderación. Para garantizar que los temas de interés se abordaran de manera conveniente y rigurosa, sin que ello supusiera un obstáculo a la espontaneidad y dinamismo, se diseñó una guía de contenidos flexible que contempló las siguientes cuestiones 
para inducir el debate: diversidad en el aula, apoyos y obstáculos a la inclusión, recursos disponibles. La duración empleada durante la recogida de la información fue distribuida en las distintas fases: Presentaciones e instrucciones de la sesión (10 minutos). Distribución y cumplimentación de la ficha de recogida de datos sociodemográficos y trayectoria profesional (15 minutos). Desarrollo de los grupos, realizados de forma simultánea y con una duración de 1 hora y 50 minutos y 2 horas y 6 minutos respectivamente. A modo de cierre, se realizó una puesta en común en la que se intercambiaron comentarios y reflexiones finales (30 minutos).

En la fase de análisis de la información, se realizaron las siguientes tareas: transcripción literal de las grabaciones, lectura preliminar de las transcripciones, elaboración de las categorías de análisis, codificación, asignación de cada fragmento a las categorías temáticas, análisis de contenido, elaboración de redes semánticas y redacción final del análisis. Para todo ello se utilizó el programa de análisis cualitativo Atlas.ti y las categorías y subcategorías de análisis finalmente creadas y utilizadas en el presente artículo fueron las siguientes (cuadro 1).

Cuadro 1

Categorías y subcategorías de análisis

\begin{tabular}{ll}
\hline Categorías & Subcategorías \\
\hline \multirow{2}{*}{ Nociones sobre la diversidad y discapacidad } & $\begin{array}{l}\text { Diversidad asociada a la discapacidad } \\
\text { Diversidad como diferencia de las capacidades humanas }\end{array}$ \\
& Curriculum formal \\
La gestión en el aula: estrategias inclusivas y & Curriculum oculto \\
resultados & Resultados académicos \\
& Implicaciones de toda la comunidad educativa \\
& Motivación/ no motivación intrínseca del profesorado \\
Comunidad educativa y su papel en la inclusión: & Implicación de las familias \\
familias, profesorado y alumnado & Familias de alumnado con diversidad funcional \\
& Beneficios de la inclusión de alumnado con NEE \\
\hline
\end{tabular}

Nota. Elaboración propia.

Los discursos fueron anonimizados y codificados para garantizar la confidencialidad requerida y a la que se comprometieron las investigadoras a través del documento de consentimiento informado. Los códigos se corresponden con Mp (Maestros de primaria), en referencia a los y las maestros/as de aula ordinaria; y Ma (Maestros de apoyo), donde se incluye tanto a maestros/as de aula de apoyo a la integración, pedagogos/ as y maestros/as con formación especializada en atención a la diversidad y auxiliares técnicos educativos.

\section{Resultados}

\subsection{La diversidad como elemento inherente al centro educativo}

Al indagar sobre la concepción de los participantes sobre la diversidad, se pone de manifiesto una dualidad conceptual. Por una parte, existe una preminencia de discursos focalizados en la vinculación de la diversidad con cuestiones meramente funcionales. Sin embargo, también tienen presencia los discursos que evidencian una concepción de la diversidad en sentido amplio, concebida como el conjunto de características individuales que diferencian a unas personas de otras. Por tanto, en el contexto de educación primaria, la diversidad para el profesorado se manifiesta, y por tanto se define, en relación a dos elementos que señalamos a continuación: a) la diferencia intrínseca al ser humano plural; y b) la amalgama de capacidades a nivel físico, sensorial y cognitivo. Nuestro análisis partirá de la concepción general (diversidad en sentido amplio) para centrarse en lo específico (diversidad funcional), si bien los discursos estuvieron desde el inicio marcados por la concepción restringida. 


\subsubsection{La diversidad entendida en su sentido más amplio, fruto de la sociedad plural y ejemplificada en elementos vinculados a la propia diferencia intrínseca entre individuos.}

Esta noción amplia de la diversidad encuentra su base en la pluralidad en las maneras de concebir la realidad, así como de relacionarnos y comportarnos entre individuos, que pone de manifiesto las diferentes posibilidades de interacción. Así, según los discursos, esta diversidad debe ser considerada hecho inherente a la sociedad y hacerse presente, por ende, en los centros educativos.

Creo que la propia personalidad ya aporta diversidad. (...) La personalidad ya está marcando una diferencia entre un individuo y otro. (...) La diversidad son los gustos, opiniones, creencias personales, ideología. (Mp2)

Pese a que el grupo identifica esta diversidad genérica, tanto los ejemplos que verbalizan como la terminología que emplean refleja la preponderancia de la noción de diversidad funcional frente a otras diversidades. Cabe clarificar que a través de las preguntas formuladas se les incitaba a reflexionar sobre la diversidad en su conjunto, sin hacer las moderadoras matices al respecto. Sin embargo, los expertos asocian la diversidad a la presencia de alumnado con diversidad funcional en centros ordinarios de educación primaria.

\subsubsection{La diversidad vinculada a las capacidades funcionales y que aparece asociada, con matices, a la discapacidad.}

En el imaginario colectivo del profesorado participante en los grupos focales prevalece, como se ha reflejado anteriormente, la idea de diversidad asociada a discapacidad o diversidad funcional, frente a la diversidad cultural, étnica, lingüística, de género, de orientación o identidad sexual o de otro tipo.

Porque la diversidad es, como estáis diciendo, las diferentes capacidades que tenemos y demás. (Ma5)

Yo considero que también hay diversidad porque cada alumno necesita que lo ayuden de diferente manera. (Ma1)

Desde esta orientación, una de las informantes induce el debate de la discapacidad versus diversidad funcional, realizando una diferenciación terminológica que, no obstante, supera la mera reflexión epistemológica sobre la nomenclatura empleada, poniendo el foco en la pertinencia de desarrollar y fomentar los talentos y aptitudes individuales desde una concepción positivista de la diferencia.

La diversidad, como estáis diciendo, son las diferentes capacidades que tenemos (...) si uno no tiene el sentido de la vista, aprovecha el sentido del oído. (Ma5)

En esta línea argumental, el profesorado insiste en identificar la escuela como parte de la sociedad plural, en la que estén presentes distintas capacidades funcionales y emocionales, en lugar de como un espacio artificial y centrado en el perfil de alumnado normotípico. Se trata, por tanto, no solo de aceptar y respetar la diversidad y la diferencia (justicia como reconocimiento), sino de ponerla en valor e incluirla, evitando posibles situaciones de desigualdad, generadoras de discriminación y, en última instancia, constitutivas de segregación en el contexto escolar. Esta inclusión implica que el sentimiento de pertenencia y la participación real del alumnado diverso (justicia como participación) sea equiparable, con independencia de sus diferencias de partida (Chiner, 2011).

En el abordaje de lo que continúa siendo un reto, cabe destacar la asociación que los/as profesionales hacen entre diversidad y ejercicio docente, reflejando la importancia que el profesorado da a su labor y a la estrategia docente como medio para atender adecuadamente la diversidad en el ámbito educativo. En este sentido, y abordándose en profundidad en el siguiente apartado, evidencian un estancamiento en la práctica docente inclusiva y una desvirtuación entre la teoría analítica, las políticas sociales implantadas y las exigencias de la práctica profesional en el aula.

\subsection{La gestión de la diversidad funcional en el aula. Resultados académicos, adaptacio- nes y estrategias de inclusión}

Respecto a la gestión de la diversidad funcional en el aula general, una de las cuestiones que resulta de mayor complejidad al profesorado es la combinación o ajuste entre la heterogeneidad en los ritmos de aprendizaje presentes en el aula y el logro de los resultados académicos exigidos. Se evidencia así, tal y como apuntan 
diversos expertos en la materia (Garzón, Calvo y Orgaz, 2016; Martínez, De Haro y Escarbajal, 2017), la pertinencia de reenfocar la perspectiva, garantizando que sea el currículo educativo el que se adapte a las necesidades y capacidades del alumnado y no a la inversa. En este sentido, los participantes del grupo se dividen entre quienes consideran que la normativa actual no permite llevar a cabo ajustes eficaces de currículo para adaptarlo a las necesidades del alumnado, y quienes estiman que esto sí es posible, pero muestran reticencias sobre la burocracia exigida para la ejecución de dichos cambios.

Los informantes refieren la falta de efectividad del sistema bajo la consideración de que, en términos generales, éste impone resultados homogéneos según área y etapa. Dicho modelo de enseñanza no consigue incorporar la diversidad de las capacidades que el alumnado posee.

Vamos a evaluar por igual a todo el mundo. Hablamos de diversidad, pero no hay diversidad en lo que luego queremos evaluar. Da igual si tú eres una máquina en matemáticas y a ti te cuesta más, da igual, te vamos a evaluar exactamente de lo mismo con la misma regla. (Ma3)

Los participantes advierten de la preeminencia de contenidos formales en el currículo frente a contenidos transversales que, si bien se recogen en el actual sistema, quedan relegados a un segundo plano en la práctica escolar debido a las exigencias curriculares impuestas. En este sentido, la mayoría coincide en la necesidad de impulsar y dar valor a este tipo de contenidos transversales, dada la disparidad y complejidad de las situaciones presentes en los centros y la necesidad de atender a la diversidad presente en las aulas. Reflexionan, además, sobre la actual sociedad orientada al éxito, en la que parecemos olvidar que el "rendimiento académico no es lo único que importa” (Ma3), en la línea de lo apuntado por autores como Braslavsky (2006) que pone de manifiesto que nos niños merecen "ser felices en la escuela” (p.87) o Verdugo (2009), que expone que "la meta o misión de la educación debe centrarse en mejorar la calidad de vida de cada alumno" (p.26). En definitiva, realizan una defensa argumentada en la importancia de trabajar en mayor medida contenidos transversales y cuestiones vinculadas al bienestar en educación primaria, denominado “currículo oculto”.

En el espectro autista, a lo mejor un niño te ha conseguido en muy poco tiempo unas habilidades sociales que, eso les cuesta la vida, iy eso no los valoramos! Y eso algo que va a marcar de por vida. (Ma3)

Hay que atender más al currículo oculto. Hay muchas cosas que se escapan de las manos de los maestros y hay situaciones por ejemplo en los recreos (...) hay que atender bastante esas conductas. (Ma4)

En relación al alumnado con diversidad funcional, reflejan la ausencia de precisión en los diagnósticos externos y en la identificación de medidas de apoyo, convirtiéndose esta falta de rigor en un elemento obstaculizador de la educación inclusiva. Demandan, así, un estudio más profundo de las necesidades del alumnado, así como mayor colaboración con los equipos de orientación y especialistas.

Yo con mi especialidad, que no se amoldan en nada con el tema de los sordos, los ciegos y los sordo-ciegos. (Ma2)

Tampoco hay acuerdo con los psicólogos muchas veces. (Mp3)

Comparten también la defensa de una aplicación flexible de los requerimientos exigidos para las adaptaciones curriculares. Así, identifican la problemática de las situaciones "limbo" que se producen cuando un alumno con NEE no llega a cumplir los requisitos establecidos por la normativa para aplicársele una adaptación significativa. En estos casos, el tutor/a solo, sin apoyos, es el que realiza (o no) cambios organizativos y pedagógicos. Teniendo en cuenta la diversidad de grado y afectación del alumnado con discapacidad, así como los múltiples factores externos que interfieren en la realidad del niño/a en cuestión, se dan situaciones en las que la ausencia de una respuesta adecuada incrementa la vulnerabilidad.

Que cuando hay una norma que diga que sí puedes hacer una adaptación significativa, sí puedes hacerlo. Pero cuando no hay, o no ha llegado a ser adaptación curricular significativa, tiene que llegar a arriba del todo. (Ma2)

Para los que piensan que el sistema otorga autonomía a los docentes y flexibilidad organizativa a los centros, el problema se encuentra en la excesiva burocracia que requieren los cambios, así como en la complejidad de trasladar los preceptos normativos en relación a la inclusión a la práctica de aula y a la organización del centro. 
Yo sí creo que el sistema educativo permite flexibilizar y permite adaptarlo; lo que pasa es que quizás estamos tan acostumbrados al "tengo este libro de texto que me marca la pauta" (...) Es mucho trabajo, porque es muy burocrático, porque hay mucho papeleo. (Mp1)

El profesorado en su totalidad, coincide en que la burocracia desincentiva la realización de adaptaciones por la vía formal por la complejidad y sobrecarga de trabajo que esto les supone.

Yo creo que el sistema educativo, que era la pregunta, sí que ofrece posibilidades, pero claro, con un trabajazo detrás impresionante. (Mp1)

La dificultad de atender a la diversidad en un sistema fuertemente ligado al logro de resultados, evaluados a través de pruebas unificadas, se refleja como motivo de preocupación por parte de nuestros participantes al no adaptarse a la pluralidad de situaciones y capacidades presentes en el aula. La importancia que dan a esta cuestión se revela en la cantidad de tiempo y argumentos englobados entorno a dicha preocupación, reflejo de la controversia, malestar y desazón entre los participantes respecto a esta cuestión.

\section{Es importante el progreso que tiene el alumnado, pero también si ves a ese niñofeliz o no. (Ma4)}

Pese a la preocupación manifestada, se observa en los participantes una alta motivación hacia el desarrollo de prácticas inclusivas, orientadas a la convivencia y bienestar socioemocional de los niños/as. Reflejan la pertinencia del desarrollo de estas actividades en el conjunto de centros y aulas, pero especialmente en aquéllas en las que hay niños/as con diversidad funcional. De entre las actuaciones impulsadas, destacan las siguientes: De detección de situaciones de rechazo y prevención de actitudes discriminatorias y/o conductas violentas; actividades que fomentan la autonomía; desarrollo de habilidades sociales; iniciativas de fomento de la diversidad y puesta en valor de la misma.

Cabe destacar, no obstante, que esta motivación se entremezcla con una visión crítica de los apoyos formales recibidos, demandando un mayor acompañamiento y asesoramiento en este camino emprendido.

No podemos creer que la responsabilidad única de la inclusión pertenece al docente. Pertenece a todo el centro educativo: como centro y como comunidad. (Mp2)

A veces los docentes nos sentimos muy solos, y además con medidas que se nos imponen, contradictoria. (Mp3)

En la figura 1, representamos gráficamente la alusión que los profesionales hacen a los distintos elementos del currículo formal, resultados académicos y currículo oculto, en lo que refiere a la gestión del aula.

Figura 1

Gestión dentro del aula

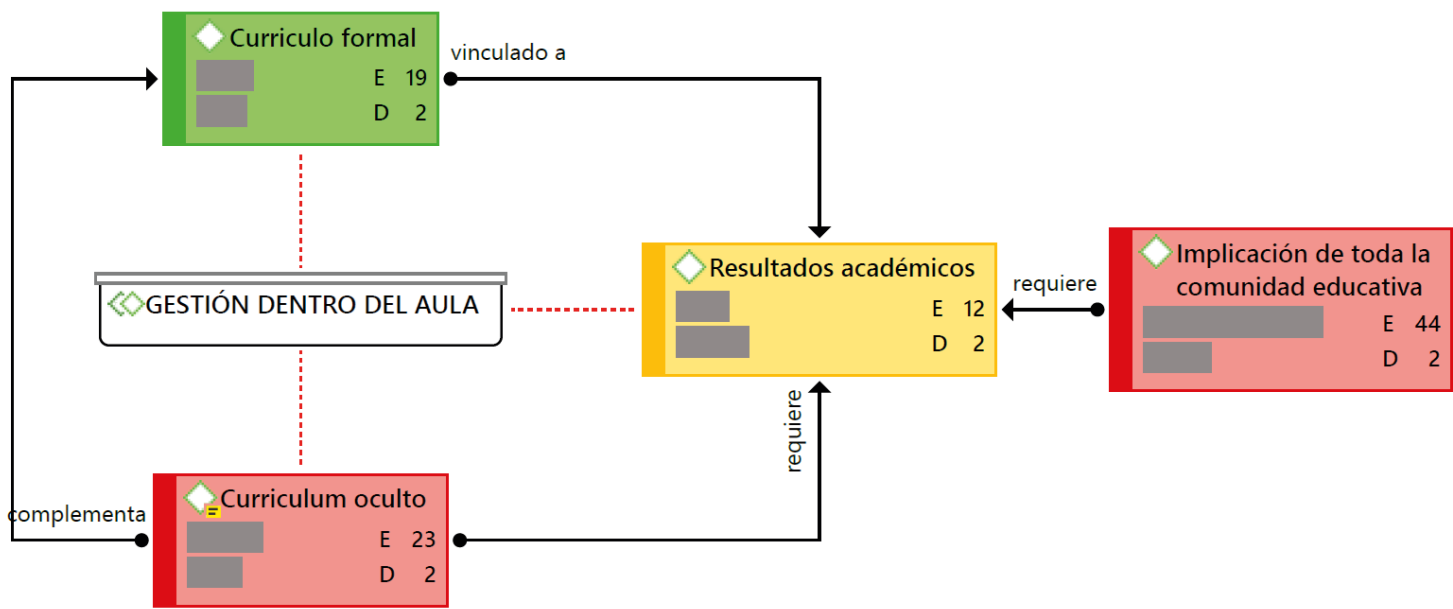

Nota. "E" hace referencia al enraizamiento (número de citas asignadas a ese código) y "D" a la densidad (número de relaciones que existen entre los códigos). Elaboración propia. 


\subsection{Comunidad educativa. Apoyos y resistencias de la inclusión de alumnado con di- versidad funcional}

La comunidad educativa, conformada por familias, equipo educativo, alumnado y comunidad/barrio, es identificada como pieza clave en la conformación de una escuela diversa donde la presencia de alumnado con diversidad funcional no sea considerada un problema. A continuación, identificamos los posicionamientos de familias, alumnado y profesorado sobre la coexistencia de alumnado con y sin diversidad funcional en el aula.

\subsection{Las demandas de los padres}

Si bien es corriente que exista diversidad entre los progenitores en relación a las expectativas escolares de sus hijos e hijas, el profesorado advierte que son frecuentes las manifestaciones de rechazo ante la presencia en el aula de alumnado con diversidad funcional o/y necesidades específicas de apoyo educativo. Del análisis de contenido encontramos que, según el profesorado, este recelo suele manifestarse de dos maneras.

Por un lado, de manera grupal, bajo la creencia de que el nivel de aprendizaje disminuye a medida que la diversidad del grupo aumenta (diversidad étnica, cultural, diversidad funcional, etc.), o percibiendo que existe una menor dedicación del profesorado en relación a sus hijos/as, consecuencia de la presencia en el aula de alumnado con NEE.

Es uno de los problemas grandes que tiene la inclusión, que se puede pensar que puede retrasar las sesiones. (Ma6)

Por otro lado, de manera individual, a través de manifestaciones de discafobia transmitidas de padres a hijos/as, por ejemplo, desincentivando a que su hijo/a comparta juego con alumnado con diversidad funcional.

Hay veces que son las familias las que le dicen, no te sientes en esta mesa que tal, cámbiamelo que lleva mucho tiempo con X (alumno con diversidad funcional) y no se va a enterar. (Ma3)

Gente que no quiere que su hijo se junte con tal... Abogando por la no diversidad. (Ma5)

El conjunto del profesorado coincide en la gravedad de estas actitudes discriminatorias y en la necesidad de su reversión. Para ello, consideran que es importante que los progenitores participen más enérgicamente de las actividades del centro educativo, aunque reconocen que es una tarea muy difícil de lograr. Tal y como exponen Calvo, Verdugo y Amor (2016), esa participación pasa por la planificación de actividades concretas orientadas a esa finalidad, en el que se determinen los tipos de participación posibles y el rol a desempeñar por las familias en las mismas.

Trabajar con las familias, hacer talleres (...), que empiecen a ver ellos que la diversidad está en todos sitios donde mires, y que es necesario ver que no van a aprender ni más ni menos, sino que todos van a aprender y a enriquecerse. (Mp1)

Para el profesorado, el conocimiento de la diversidad, el respeto y valor de la misma, son cuestiones que se trasmiten al alumnado pero que, si no se refuerzan en casa, o peor aún, si se contradicen, no pueden consolidarse como elementos protectores frente a la discriminación o segregación de la minoría en el centro educativo. De ahí la importancia de involucrar a las familias para lograr un avance sostenible y real.

Debería existir una conexión (...) Para que los padres participen, para que les informen a ellos sobre lo que es la inclusión, para que no lo vean con miedo, sino como una ventaja para sus hijos. (Mp4)

En lo que respecta a las familias con alumnado con diversidad funcional, para el profesorado éstas son quienes perciben las consecuencias directas de las actitudes de rechazo tanto del sistema como de la sociedad en relación a sus hijos/as. Valoran positivamente la agrupación colectiva de padres y madres de alumnado en Asociaciones y las reclamaciones conjuntas que realizan al sistema educativo para exigir la efectividad de la inclusión. Los participantes reflejan que este tipo de presiones ha sido el motor para la incorporación al sistema de muchas figuras profesionales y recursos educativos anteriormente inexistentes.

Los padres son una fuerza de peso muy grande. (Mp1) 
En relación al centro, a los padres de alumnado con diversidad funcional les preocupa la conveniente respuesta de cuidados de salud que pueda requerir su hijo/a en horario escolar y/ actividades extraescolares. Se muestran, así, exigentes hacia el profesorado, especialmente el tutor/a, al respecto de la implicación en las necesidades educativas específicas y sociabilidad de su hijo/a con el resto de alumnado.

Nos hemos ido de viaje de estudios y tenemos un niño asperger (...) cuando nos hemos juntado con la madre, nos dijo que estuviéramos más pendientes de las rutinas, las comidas, para dormir (...). Y hemos ido 80 alumnos y ha sido, bueno tú te centras en él y el resto en los demás. (Ma2)

En ocasiones, el profesorado percibe una actitud de sobreprotección de los progenitores hacia los hijos/as, aunque matiza que ésta no está únicamente motivada por la situación de discapacidad. Apuntan que la sobreprotección parental es contraproducente, actuando como un elemento obstaculizador más en el desempeño personal y educativo de los niños/as, en lugar de contribuir a él.

\section{Algunos tienden a sobreproteger y a creer que su hijo va a poder menos de lo que realmente puede. (Mp2) \\ A veces los sobreprotegen, los meten en una burbuja, yo por ejemplo he visto que el chiquillo entra en el centro y es una persona, y sale del centro y no lo conozco. (Ma7)}

El profesorado de aula ordinaria con alumnado con y sin discapacidad convive con las demandas de unas familias y otras en relación el bienestar de sus hijos/as. Sin embargo, lo que más preocupa a los y las docentes que participan en nuestro grupo focal es la poca implicación de los progenitores de alumnado sin diversidad funcional en la actividad del centro escolar.

\subsection{Actitudes del profesorado y escuela en relación a la inclusión}

El trabajo de inclusión de alumnado diverso dentro de un centro educativo necesita de coordinación y colaboración entre compañeros para ser efectivo. Sin embargo, en el día a día surgen inconvenientes de diversa índole que obstaculizan de manera directa o indirecta la inclusión. Por ejemplo, los informantes verbalizan que el celo profesional entre compañeros/as es más común de lo esperado y deseable en el contexto de educación primaria y actúa como elemento limitante a la inclusión. A modo de autocrítica, algunos reconocen que evitan compartir información para evadir el cuestionamiento o intromisión de terceras personas en su trabajo. A pesar de las consecuencias negativas para el alumnado que esto puede tener, y de las que son conscientes, afirman que en muchas ocasiones han rehusado compartir cierta información con sus compañeros, sobre todo al inicio de su actividad profesional, influenciados, entre otras, por la competitividad presente en la formación académica y procesos selectivos previos a la incorporación en el centro educativo.

Muchas veces no sabemos ni tampoco nos interesa trabajar en equipo. (Ma8)

Cuanto piden algún informe, se miran entre ellos o ves que lo revisan antes de dártelo (...) Yyo no voy a decir si lo tienen bien o mal, yo lo único que quiero es trabajar. (Ma2)

Además, desde el punto de vista de los participantes, dentro de la plantilla docente, existe un porcentaje del profesorado que está muy poco o nada implicado en la inclusión de alumnado con diversidad funcional en el aula, reflejado en una dedicación mínima y reducida a las exigencias impuestas por el sistema, llegando a producirse en ocasiones dejación de funciones básicas.

A veces que te encuentras aulas en los que hay profesores que no atienden a ese alumnado (diversidad funcional), es como si fuera un estorbo para ellos. (Mp4)

Yo he oído mucho eso, si tienes tres niños con diversidad funcional, yo que sé, un niño sordo y dos con autismo, ya esa clase ya no llega. Y el primero el tutor, tú mismo sin querer haces Uf, es que la clase que me ha tocado. (Mp3)

En el otro extremo, se encuentra el profesorado implicado y comprometido con la inclusión o, como ellos/ as refieren, el profesorado que llevan puestas las gafas de la inclusión. Esta motivación intrínseca hacia la inclusión, les lleva a involucrarse activamente en la gestión de la diversidad o incluso impulsar actividades extracurriculares dentro del centro. Sin embargo, el profesorado que se identifica con este tipo de perfil, explica que sin el apoyo del resto del profesorado resulta muy difícil llevar a cabo cambios o avances en 
relación a este tema. Aluden así a la importancia de la coordinación, colaboración y trabajo en equipo, evitándose asíla sensación de que,

¡vuelves a ser tú, remando sola! (Mp3)

Yo diría colaboración, pero claro, eso no es algo que es tan personal... Porque, por ejemplo, nosotras este año hicimos charlas para que todo el personal conociese el autismo, entonces hubo buena respuesta. Pero no siempre accedes a determinadas personas (refiriéndose a compañeros) as). (Mp1)

Los/as participantes de nuestros grupos coinciden en que, para lograr la inclusión del alumnado con diversidad funcional en un centro ordinario, no solo el profesorado debe estar comprometido con esto de forma individual, sino que resulta imprescindible que el equipo también lo esté. Apuestan así, por un equipo capaz de dedicar sus esfuerzos a realizar un estudio profundo del centro, del entorno, de las necesidades del alumnado, y de adaptar el currículo a la realidad presente en los distintos niveles.

\subsection{El alumnado con diversidad funcional: Principal beneficiario, pero no único}

Los/as informantes refieren al mismo tiempo la complejidad y beneficios de la inclusión educativa. Para la mayoría de ellos, el esfuerzo durante el curso compensa porque perciben que el impacto positivo para el alumnado con y sin diversidad funcional. En relación a quién beneficia las prácticas inclusivas de la escuela, el profesorado indica lo siguiente:

\section{Yo diría que, al menor, el alumno (refiriéndose al alumnado con diversidad funcional). (Ma3) \\ Yo pienso que beneficia también muchísimo al resto de alumnos. (Mp3)}

Los prejuicios existentes en la comunidad contradicen lo expresado por el profesorado con más experiencia, quienes consideran que los recursos que apoyan el proceso educativo del alumnado con NEAE tienen impactos positivos en la totalidad del alumnado. A nivel pedagógico, por ejemplo, señalan que recursos como el Intérprete de lengua de signos española (ILSE) apoya el aprendizaje no sólo del alumnado sordo, sino también del resto de alumnado porque complementa el lenguaje verbal. Especialmente para el alumnado con escaso manejo del idioma o con dificultades de aprendizaje leves, este tipo de recurso u otros como el Mediador Comunicativo, resultan de gran apoyo.

Resulta que en mis clases aprendían a leer y escribir muy rápido y yo me di cuenta que lo que habia alli era un apoyo más (en referencia a un aula ordinaria con un alumno sordo que tenía apoyo de un/A ILSE). (Mp2)

Los beneficios para todo el alumnado, también son notables cuando el profesorado se esfuerza por generar en el aula entornos de aprendizaje con mayor potencial pedagógico.

Descubrí los articulemas. Un abecedario hecho en cartón, y trabajábamos con los sonidos en lengua de signos, articulema y letra. (Mp3)

Por otra parte, las adaptaciones físicas que garantizan el acceso y movilidad en condiciones de seguridad del alumnado con discapacidad en el centro educativo, no solo benefician al alumnado y/o profesorado con movilidad reducida, sino al conjunto de la comunidad educativa. Lo mismo ocurre con otro tipo de recursos que favorecen el acceso universal a la educación.

Por ejemplo, la eliminación de barreras, muros, puertas estrechas, que eso al final beneficia a todo el mundo. (Ma4)

La rampa, tu no llevas silla de ruedas, pero seguro que si vas con una maleta la usas. Pues la lengua de signos es lo mismo, si hay interprete, las ideas te van a quedar más por su signo, y en tu casa, cuando estás estudiando te vuelve el signo y es una forma de recuperar información. Es un recurso súper útil tengas o no tengas discapacidad auditiva. (Mp2)

En definitiva, los recursos específicos de apoyo dirigidos alumnado con discapacidad resultan imprescindibles para el alumnado con NEE y convenientes para la totalidad del alumnado, resultando importante hacer consciente a los distintos grupos que conforman el entorno educativo de que los beneficios de la inclusión no solo lo son para el alumnado con diversidad funcional, sino para el conjunto de la comunidad educativa. 
En la figura 2 se representa gráficamente los actores y elementos presentes en la comunidad educativa que influyen en que la conformación de una escuela inclusiva.

Figura 2

Actores y elementos presentes en la escuela inclusiva

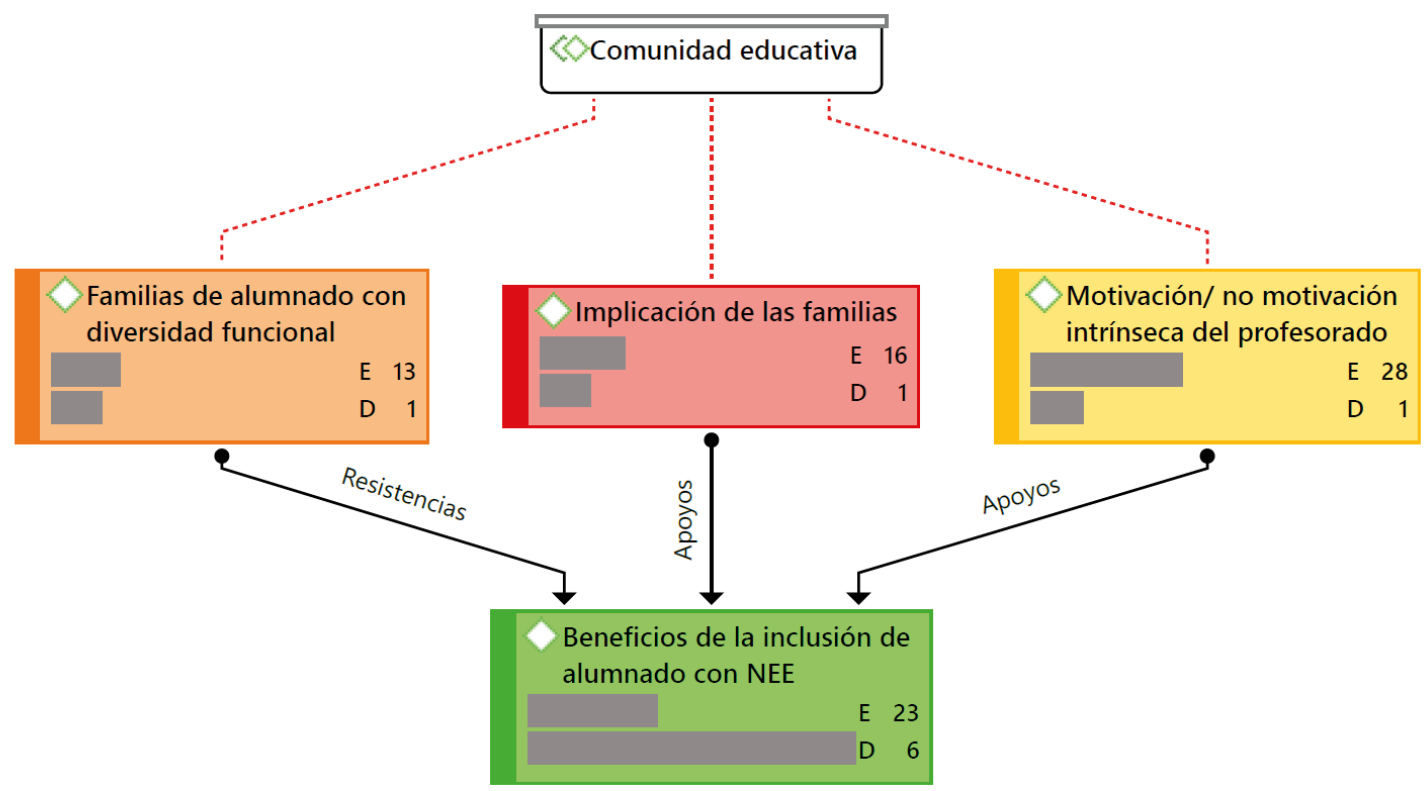

Nota. Elaboración propia.

\section{Discusión y conclusiones}

La importancia de la educación inclusiva ha sido constatada en los distintos ámbitos formales, siendo definida de manera polisémica por la Convención Internacional de Personas con Discapacidad (Naciones Unidas, 2006) como un derecho humano, un medio, un principio y un proceso. Sin embargo, la mayoría de los sistemas abordan el reto de la inclusión focalizando la atención en el derecho, si bien el alcance del mismo pasa por lograr que la excelencia o calidad educativa alcance, a través de la equidad, a todo el alumnado. Así nuestros participantes, en línea con lo apuntado por estudios previos, defienden la pertinencia no solo de la educación para todas las personas en centros ordinarios, sino más bien de una educación de calidad y excelencia para todas las personas en entornos inclusivos, en los que dicha inclusión resulte beneficiosa tanto para unos como para otros.

Este reto precisa de la implantación de una serie de cambios a nivel de macrosistema -políticas educativas-, mesosistema -organización institucional-y microsistema -prácticas educativas- (Verdugo, 2009), con implicaciones a nivel estructural, de actitudes, de formación, de flexibilización del CV y de avance en la concepción de la educación como una organización abierta a la comunidad con una notoria incidencia en aspectos críticos para el desarrollo humano. Uno de los aspectos más reseñados en nuestros grupos focales, es la importancia de no orientar los objetivos únicamente al rendimiento académico, sino también a contenidos transversales y retos de bienestar psicosocial. Así, se pone de manifiesto la preponderancia en el sistema actual de valores tendentes a la competitividad, prosperidad y rendimiento (principios académicos/económicos), frente a la inclusión de la diversidad (principios sociales/de justicia social), que se refleja en la priorización del currículo formal frente al "oculto" y en la homogeneización de resultados exigidos según área y etapa. Identifican así una infrarrepresentación y escasa puesta en valor tanto de los contenidos transversales como de las identidades normoatípicas, que conduce a la segregación académica, y que solo puede superarse a partir de principios inclusivos y participativos. En este sentido, se observa por parte de los participantes una motivación a la inclusión, puesta de manifiesto a través del desarrollo de actividades diversas, si bien demandan un mayor acompañamiento por parte de instancias superiores. 
Además, los/as participantes identifican una falta de implicación en la consolidación de entornos escolares inclusivos por parte de un sector considerable de profesionales educativos. El prejuicio de esta realidad resulta incuestionable, más cuando estudios previos (p. ej., Chiner, 2011) han apuntado a las actitudes del profesorado como determinantes de la acción e indicadores de buenas prácticas (o lo contrario). Sola (1997) señala como elementos impulsores de actitudes positivas hacia la inclusión educativa la formación coherente y planificada, tanto inicial como continuada. A su vez, Sales, Moliner y Sanchiz (1997) apuntan que esa formación será la clave para empoderar a los docentes con actitudes y competencias en la materia a buscar, reflexionar y consensuar con los agentes implicados alternativas que permitan a la totalidad del alumnado acceder al máximo de sus capacidades y al desarrollo personal y social. Además, se constata la necesidad de consolidar una red colaborativa, conformada por el conjunto de la comunidad educativa, en la que se avance en la materia a través de la implicación y convivencia de todas las partes involucradas.

Cabe destacar que, si bien se induce al debate sobre la diversidad, en sentido amplio, y la atención a las necesidades específicas de apoyo educativo, también desde una perspectiva holística, los discursos y argumentos se focalizan de manera espontánea hacia la diversidad funcional y las necesidades educativas especiales, habiendo una sobrerrepresentación de estas necesidades frente a otras. Por último, entre las limitaciones que podía presentar nuestra investigación, se encontraba la creciente aceptación social de la inclusión educativa, que podía influir en las respuestas de nuestros/as participantes por deseabilidad social. Sin embargo, la consistencia de los argumentos esgrimidos, nos lleva a deducir un compromiso real hacia la inclusión. Por otra parte, y considerando la imposibilidad de hacer generalizaciones a partir de un estudio con enfoque cualitativo, se propone avanzar en esta investigación a partir del estudio de caso de un centro educativo representativo de la provincia, con el objeto de analizar la inclusión educativa de la diversidad funcional en los niveles macrosistema, mesosistema y microsistema. Dicha aproximación permitiría analizar la planificación de estudios, la dotación y gestión de recursos, los contenidos del currículum y las prácticas docentes, relacionadas con la diversidad, equidad e inclusión de todas las personas, que permita plantear propuestas concretas de mejora.

Por tanto, cabe destacar las siguientes conclusiones principales de la presente investigación:

- Tendencia a conectar la diversidad con la diversidad funcional, con una mayor presencia de discursos de ésta frente a otras diversidades presentes en el aula.

- Importancia de visibilizar los beneficios de la inclusión educativa para el conjunto de la comunidad educativa y ciudadanía general.

- Reclamo de una mayor implicación hacia la inclusión y demanda de un mayor acompañamiento y apoyo a aquellos/as maestros/as que presentan predisposición positiva y motivación al desarrollo de estrategias de educación inclusiva.

- Necesidad de reorientar los objetivos educativos, otorgando más peso a contenidos transversales frente a los meramente académicos.

\section{Referencias}

Araque, N. y Barrio J. L. (2010). Atención a la diversidad y desarrollo de procesos educativos inclusivos. Prisma Social, 4,1-37.

Arnaiz, P. (2012). Escuelas eficaces e inclusivas: cómo favorecer su desarrollo. Educatio Siglo XXI, 30(1), 25 - 44.

Braslavsky, C. (2006). Diez factores para una educación de calidad para todos en el siglo XXI. REICE. Revista Iberoamericana sobre Calidad, Eficacia y Cambio en Educación, 4(2), 84-101.

Calvo, M. I., Verdugo, M. A. y Amor, A. M. (2016). La participación familiar es un requisito imprescindible para una escuela inclusiva. Revista Latinoamericana de Educación Inclusiva, 10(1), 99-113.

https://doi.org/10.4067/S0718-73782016000100006 
Chiner, E. (2011). Las percepciones y actitudes del profesorado hacia la inclusión del alumnado con necesidades educativas especiales como indicadores del uso de prácticas educativas inclusivas en el aula (Tesis Doctoral). Universidad de Alicante, España.

Coll, C., Pozo, J. I., Sarabia, B. y Valls, E. (1994). Los contenidos en la reforma: Enseñanza y aprendizaje de conceptos, procedimientos y actitudes. Santillana.

Díaz, A. (2006). La educación en valores: Avatares del currículum formal, oculto y los temas transversales. Revista Electrónica de Investigación Educativa, 8(1), 1-15.

Dussan, C. P. (2010). Educación inclusiva: Un modelo de educación para todos. ISEES: Inclusión Social y Equidad en la Educación Superior, 8, 73-84.

Escobar, J. y Bonilla-Jiménez, F. I. (2017). Grupos focales: Una guía conceptual y metodológica. Cuadernos Hispanoamericanos de Psicología, 9, 1, 51-67

Fraser, N. (2008). Escalas de justicia. Herder.

Fraser, N. y Honneth, A. (2005). ¿Redistribución o reconocimiento? Morata.

García-Domingo, M., Amezcua, T. y Fuentes, V. (2019). El reto de la educación inclusiva: Elementos implicados y propuestas de mejora. Prisma Social, 27(4), 40-64.

Garzón, P., Calvo, I. y Orgaz, B. (2016). Inclusión educativa. Actitudes y estrategias del profesorado. Revista Española de Discapacidad, 4(2), 25-45.

https://doi.org/10.5569/2340-5104.04.02.02

Luque-Parra, D. y Luque-Rojas, M. (2013). Necesidades específicas de apoyo educativo del alumnado con discapacidades sensorial y motora. Summa psicológica UST, 10(2), 57-72.

https://doi.org/10.18774/448x.2013.10.141

Martínez, R., De Haro, R. y Escarbajal, A. (2017). Una aproximación a la educación inclusiva en España. Revista de Educación Inclusiva, 3(1), 149-164.

Merle, P. (2012). La ségrégation scolaire. La Découverte.

Murillo, F. J. y Duk, C. (2016). Segregación escolar e inclusión. Revista Latinoamericana de Educación Inclusiva, 10(2), $11-13$.

https://doi.org/10.4067/S0718-73782016000200001

Murillo, F. J. y Hernández, R. (2011). Hacia un concepto de justicia social. REICE. Revista Iberoamericana sobre Calidad, Eficacia y Cambio en Educación, 9(4), 7-23.

Murillo, F. J. y Martínez-Garrido, C. (2017). Estimación de la magnitud de la segregación escolar en América Latina. Magis: Revista Internacional de Investigación en Educación, 9(19), 11-30.

https://doi.org/10.11144/Javeriana.m9-19.emse

Murillo, F. J., Martínez-Garrido, C. y Belavi, G. (2017). Segregación escolar por origen nacional en España. OBETS. Revista de Ciencias Sociales, 12(2), 395-423. https://doi.org/10.14198/OBETS2017.12.2.04

Naciones Unidas. (2006). Convención internacional sobre los derechos de las personas con discapacidad. http://www.un.org/esa/socdev/enable/documents/tccconvs.pdf

Nussbaum, M. (2012). Crear capacidades: Propuesta para el desarrollo humano. Paidós. https://doi.org/10.2307/j.ctvt7x63g

Rubia, F. A. (2013). La segregación escolar en nuestro sistema educativo. Forum Aragón: Revista Digital de FEAE-Aragón sobre organización y gestión educativa, 10, 47-52.

Sales, M.A., Moliner M.O. y Sanchiz, M.L (2001). Actitudes hacia la atención a la diversidad en la formación inicial del profesorado. Revista Electrónica Interuniversitaria de Formación del Profesorado, 4(2), 1-7.

Sen, A. (2010). La idea de la justicia. Taurus. 
Sola, T. (1997). La formación inicial y su incidencia en la educación especial. En A. Sánchez y J. A Torres (Coords.), Educación especial I Una perspectiva curricular, organizativa y profesional (pp. 23-34). Editorial Pirámide.

Rawls, J. (1971). A theory of justice. Harvard University Press

Rawls, J. (2002). La justicia como equidad. Tecnos

UNESCO. (1994). Final report: World conference on special needs education: Access and quality. UNESCO.

Verdugo, M. A. (2009). El cambio educativo desde una perspectiva de calidad de vida. Revista de Educación, 349, 23-43.

Young, I. M. (2000). La justicia y la política de la diferencia. Cátedra.

Young, I. M. (2002). Inclusion and democracy. Oxford University Press.

https://doi.org/10.1093/0198297556.001.0001

\section{Breve CV de las autoras}

\section{Virginia Fuentes Gutiérrez}

Doctora en Trabajo Social. Trabajadora Social, Licenciada en Ciencias del Trabajo. Profesora del Departamento de Psicología, Área de Trabajo Social de la Universidad de Jaén, profesora del Máster en Dependencia e Igualdad en la Autonomía Personal de la Universidad de Jaén. Ha formado parte del equipo de investigación de proyectos sobre las temáticas: Educación Inclusiva, inclusión socio-educativa, atención a la discapacidad en la Educación Superior, entre otros. En algunos de estos proyectos, ha ocupado el rol de Investigadora Principal. Los resultados de dichas investigaciones han sido publicados en libros, artículos en revistas científicas nacionales e internacionales y ponencias o comunicaciones en Congresos de ámbito nacional e internacional. Email: vfuentes@ujaen.es

ORCID ID: https://orcid.org/0000-0001-6876-067X

\section{Marta García-Domingo}

Doctora en Trabajo Social. Trabajadora Social, Master en Dependencia e Igualdad en la Autonomía Personal y Master en Accesibilidad Universal y Diseño para todas las Personas. Profesora del Departamento de Psicología, Área de Trabajo Social de la Universidad de Jaén, así como de los Másteres anteriormente mencionados. Ha participado en diversos proyectos de investigación relacionados con la atención a la diversidad y la inclusión socioeducativa, así como difundido y publicado los resultados de dichas investigaciones en congresos internacionales y revistas de prestigio. Email: mgdoming@ujaen.es

ORCID ID: https://orcid.org/0000-0002-8597-5549

\section{Patricia Amezcua Aguilar}

Graduada en Educación Primaria con especialidad en Pedagogía Terapéutica y Máster en Psicopedagogía. Técnico Superior en Interpretación de Lengua de Signos. Es experta en las dificultades de aprendizaje relacionadas con la diversidad funcional sensorial. Orientadora Laboral y docente de Formación Profesional para el Empleo. Docente de Lengua de Signos. Sus intereses de investigación giran en torno a las necesidades educativas especiales en Educación Primaria, metodologías inclusivas y la diversidad funcional. Email: patricia.ilse@hotmail.com

ORCID ID: https://orcid.org/0000-0001-8445-3725

\section{Teresa Amezcua Aguilar}

Doctora en Cuidados Integrales y Servicios Sociales, Trabajadora social, Máster en Dependencia e Igualdad en la Autonomía Personal y Gerontóloga Social. Profesora del Departamento de Psicología, Área de Trabajo Social de la Universidad de Jaén. Profesora del Programa Universitario de Inclusión Educativa 
“Univerdi” y del Máster en Accesibilidad Universal de la Universidad de Jaén. Es también Intérprete de Lengua de Signos y Guía Intérprete de personas sordas y sordociegas. Sus intereses de investigación giran en torno a los procesos inclusión/exclusión social, la diversidad funcional, discapacidad, envejecimiento, accesibilidad y participación social. Email: mamezcua@ujaen.es

ORCID ID: https://orcid.org/0000-0002-8128-8717 